\title{
Quantifying the psychological value of goal achievement
}

\author{
Timothy Ballard ${ }^{1} \cdot$ Simon Farrell ${ }^{2} \cdot$ Andrew Neal $^{1}$
}

Published online: 6 July 2017

(C) Psychonomic Society, Inc. 2017

\begin{abstract}
It is often assumed that people put forth the least amount of effort necessary to obtain a reward. This assumption is consistent with so-called "rational" economic models of behavior. Yet these models rarely take into account the motivating effects of goals, which may lead to departures from objective reward maximizing behavior. We present an experiment in which people make a series of prioritization decisions whilst pursuing two approach or avoidance goals. Participants were rewarded $\$ 10$ if they achieved both goals on a randomly selected trial, and either $\$ 0, \$ 2.50, \$ 5$, $\$ 7.50$, or $\$ 10$ if they achieved only one. Bayesian parameter estimation was used to examine the subjective values that people placed on various goal achievement outcomes. The results suggested that people often discounted the achievement of the first goal, relative to a reward maximizing model, particularly when pursuing avoidance goals. These results were most evident among participants who could obtain the full reward after achieving just one goal, yet behaved as if achieving one goal was only half as valuable as achieving both. Our findings question the notion that people put forth the least amount of effort required to obtain a reward. They suggest that when tasks have explicit goals, people may even sacrifice financial reward to achieve the goals.
\end{abstract}

Electronic supplementary material The online version of this article (doi:10.3758/s13423-017-1329-1) contains supplementary material, which is available to authorized users.

Timothy Ballard

t.ballard@uq.edu.au

1 The University of Queensland, Brisbane, Queensland, Australia

2 The University of Western Australia, Perth, Australia
Keywords Reward · Goals · Decision-making · Bayesian modeling

\section{Quantifying the psychological value of goal achievement}

People often face situations in which they must strategically allocate resources in dynamic environments in order to attain rewards and avoid punishment. For example, most people have more than one goal they need to achieve at work, and have to decide how they will allocate their time and effort in order to achieve those goals. Retail workers face this dilemma on a daily basis as they decide how to best allocate their time in order to reach sales targets, whilst also providing good customer service. As academics, we face the same dilemma as we juggle competing demands for research, teaching, and administration. Resources such as time and effort are limited. For this reason, it is typically assumed that there is a cost to the allocation of time and effort, and that people will attempt to maximize the utility of their efforts, trying to obtain the most reward for the least amount of work (Botvinick \& Braver, 2015; Kool \& Botvinick, 2014; Kool et al., 2010; Shenhav et al., 2013; Westbrook et al., 2013). Consistent with this view, people often demonstrate a willingness to accept smaller monetary rewards for tasks that require less work, a phenomenon known as effort discounting (Botvinick et al., 2009; Chong et al., 2015; Libedinsky et al., 2013). These findings are consistent with so-called "rational" economic models, which assume that behavior is guided by the desire to maximize expected financial rewards (e.g., James, 1993; Smith, 1976, 1982).

One problem with the reward-maximization perspective is that it fails to take into account the influence that goals have on behavior. There is a long history of research 
demonstrating that people are motivated by goals (Heath et al., 1999; Tubbs, 1986; Wang \& Johnson, 2012), and derive pleasure from achieving them (Brunstein, 1993; Koo \& Fishbach, 2008; Sheldon \& Kasser, 1998). In particular, people have been found to be more motivated by goals that involve avoiding undesirable outcomes (i.e., avoidance goals) than by those that involve achieving desired ones (i.e., approach goals; Baumeister et al. 2001). This research stands in contrast to rational economic models of behavior because it suggests that people may forgo financial rewards to increase their chances at goal achievement. As a result, people may expend more effort in return for less financial reward.

There is a growing body of literature examining how people allocate time and effort when pursuing multiple goals, a process referred to as multiple-goal pursuit (for reviews see Lord et al., 2010; Neal et al., 2017). A typical paradigm in this literature involves participants making a series of prioritization decisions whilst simultaneously working on two tasks. These studies have shown that people generally allocate time and effort on the basis of need (e.g., Louro et al., 2007). All else being equal, people tend to prioritize the goal that is more difficult at that point in time (Schmidt \& DeShon, 2007; Unsworth et al., 2009), because difficulty signals to the individual that the goal requires attention or resources. However, this tendency depends on a number of factors. For example, if the more difficult goal is perceived as not achievable, people will abandon that goal and instead focus on the goal that is easier to achieve in an effort to maximize the likelihood of achieving at least one goal (Schmidt \& Dolis, 2009; Vancouver et al., 2010).

Resource allocation also depends on the type of goals people pursue (Ballard et al., 2016; Ballard et al., 2016). The tendency to prioritize the more difficult goal is stronger when pursuing avoidance goals than approach goals (Ballard et al., 2016; Schmidt et al., 2009). Using a paradigm in which goals were equally incentivized, Ballard et al. (2016) examined the extent to which resource allocation decisions under approach and avoidance maximized the expected rewards from goal achievement. The reward-maximizing model in this task tended to prioritize the more difficult goal when it was likely that both goals could be achieved, and the easier goal when it was unlikely that both goals could be achieved. The results showed departures from reward-maximization that were consistent with prospect theory (Kahneman and Tversky, 1979), which generally predicts risk aversion in the domain of gains and risk seeking in the domain of losses. When pursuing two avoidance goals (i.e., when threatened with "loss"), people were more likely than the reward-maximizing model to prioritize the more difficult goal. This behavior is riskseeking because it increases the likelihood of achieving both goals, but also increased the likelihood of failing to achieve either goal. When pursuing two approach goals (i.e., when they had the potential to "gain"), people were more likely than the reward-maximizing model to prioritize the easier goal. This behavior is risk-averse because it increases the likelihood of achieving one goal, but decreases the likelihood of achieving both. This research therefore suggests that people depart from expected-reward maximization when pursuing goals, and that the nature of this departure depends on whether goals are approach or avoidance.

One limitation of studies of multiple-goal pursuit (e.g., Ballard et al., 2016; Schmidt \& DeShon, 2007) is that incentivizing goals equally confounds monetary reward with goal achievement. When the same incentive can be obtained for each goal achieved, the behavior that maximizes expected reward is identical to the behavior that maximizes goal achievement. This makes it impossible to determine the extent to which the value placed on various outcomes is attributable to the psychological value of goal achievement or the associated financial reward.

We attempt to disentangle the psychological value of goal achievement itself from the value brought on by anticipated financial rewards. If there is psychological value associated with goal achievement, people should discount the value of attaining only one goal relative to the value of obtaining both. As a result, people should sacrifice financial reward in order to achieve goals. We present an experiment in which people made a series of prioritization decisions whilst pursuing two approach or two avoidance goals. Participants could manage these goals using one of two strategies. Prioritizing the more difficult goal at a given point in time made it more likely that both goals would be achieved, but also more likely that neither would be achieved. Prioritizing the easier goal made it more likely that one goal would be achieved, but less likely that both would. A critical feature of the experiment was the manipulation of the incentive associated with achieving the first goal, relative to achieving both goals. When the reward from achieving the first goal was relatively small, attempting to achieve both goals by prioritizing the more difficult goal maximized the expected reward. When the reward from achieving the first goal was larger, prioritizing the more difficult goal reduced the expected reward. We examine whether people discount the value of achieving the first goal by assessing the extent to which they attempt to achieve both goals, even when doing so reduces the expected reward. We then attempt to quantify the extent of this discounting using a Bayesian parameter estimation technique within a dynamic programming model. The result is a descriptive model that captures participants' subjective value functions for various goal achievement outcomes. We then compare these subjective value functions with the reward-maximizing functions in order to examine the extent 
to which people discount the value of achieving the first goal.

\section{Methods}

\section{Participants}

The sample consisted of 81 undergraduate psychology students at the University of Western Australia who participated for course credit. Due to a technical error, age and gender information was not recorded. A pilot study that sampled from the same participant population had $56 \%$ female participants and a mean age of $19.79(\mathrm{SD}=2.79)$.

\section{Experimental paradigm}

The experiment used a multiple-goal pursuit task that was broken down into a series of trials. Participants were given two goals for each trial. The first goal was to accumulate or maintain a certain number of blue points. The second goal was to accumulate or maintain a certain number of green points. In each trial, the participant made a series of decisions between two actions: Option A and Option B (see Fig. 1). Each action offered an $80 \%$ chance of success in making progress (i.e., gaining points for an approach goal/not losing points for an avoidance goal) on one goal and, independently, a $20 \%$ chance of success in making progress on the other goal. Thus, each action forced the participant to prioritize one goal at the expense of the other. Participants selected an option by pressing the left or right arrow key, and received immediate feedback about the decision outcome. Participants were then prompted to press the space bar, after which the scores would update and participants would be prompted to make the next decision. Participants' progress through the series of decisions was displayed on the screen and the trial ceased after a pre-specified number of decisions had been made.

\section{Manipulations}

The experiment used a 2 (goal frame) x 5 (incentive structure) $x 16$ (starting position) factorial design. Each of these manipulations is explained below.

\section{Goal frame}

We manipulated goal frame within participants across two levels: approach and avoidance. In the approach condition, the participants had two approach goals: to a) "achieve a score of 10 blue points or more" and b) "achieve a score of 10 green points or more". Participants began the trial with fewer points than required. Each time the selected action was successful with respect to a particular goal, the participant would gain one point of that color. When the action was unsuccessful, they would not gain any points for that color. Thus, each action had four possible outcomes: 1) +1 point for each color (i.e., an action that is successful with respect to both goals), 2) +1 blue points and +0 green points, 3$)+1$ green points and +0 blue points and, 4) +0 points for each color (i.e., an action that is unsuccessful with respect to both goals).

In the avoidance condition, the participants' two avoidance goals were to a) 'avoid a score of 9 blue points or less' and b) 'avoid a score of 9 green points or less'. Participants began the trial with more points than required. Each time the selected action was successful with respect to a particular goal, the participant would prevent the loss of points of that color. When the action was unsuccessful, they would lose one point for that color. The four possible outcomes in the avoidance condition were 1) -1 point for each color (i.e., an action that is unsuccessful with respect to both goals), 2) -1 blue points and -0 green points, 3) -1 green points and -0 blue points and, 4) -0 points for each color (i.e., an action that is successful with respect to both goals). In both the approach and avoidance conditions, a goal would be achieved if the participant had a 10 or more points of that color at the end of the trial.

\section{Incentive structure}

In this task, participants were incentivized for their performance on a single trial that was randomly selected by the computer at the end of the experiment. If participants achieved both goals on the selected trial, they would always receive $\$ 10$. If participants achieved only one goal on the incentivized trial, they would receive $\$ 0, \$ 2.50, \$ 5, \$ 7.50$, or $\$ 10$ (manipulated between participants). Accordingly, the incentive manipulation determined how much of the reward could be obtained from achieving a single goal. When the incentive for achieving only one goal was $\$ 0$, participants could not obtain any money unless they achieved both goals. When the incentive for achieving only one goal was $\$ 10$, participants could obtain the full amount from achieving only one goal, and could not gain any additional money for achieving the second goal.

\section{Starting position}

To keep the task engaging, we varied the amount of points the participant had at the start of each trial, and the number of decisions in each trial. In total, there were 32 unique trial configurations (16 for approach and 16 for avoidance, see Appendix). The number of points that participants started with ranged from 4 to 9 in the approach condition, and 10 to 14 in the avoidance condition. The number 
of decisions in each trial ranged from 2 to 6 . The trials were configured so that difficulty was held constant across approach and avoidance conditions.

\section{Procedure}

Participants performed the task on computers in an experimental laboratory. Participants were first presented with instructions (see Supplementary Materials for full instructions), where they were told the incentive structure relevant to their condition. They then completed two practice trials (one approach and one avoidance, neither were incentivized). Participants then completed 5 blocks of 32 trials each. Each block included all 16 unique trial configurations for the approach and avoidance conditions presented in random order. Thus, each participant experienced each unique configuration 5 times, and made 770 decisions in total. Before each trial, participants were reminded of the incentive structure for the experimental task. Specifically, they were told "If you achieve both goals and fail neither on the randomly selected trial, you will walk away with an extra $\$ 10$. If you achieve one goal and fail one on the randomly selected trial, you will walk away with an extra \$X. If you achieve neither goal and fail both on the randomly selected trial, you will walk away with no extra money." In these instructions, $X$ was either $0,2.50,5,7.50$, or 10 depending on the level of the incentive manipulation.

Participants were also presented with information about the goals prior to each trial. The participants' goals, scores, number of decisions remaining, and the probabilities that each action would gain or lose points were displayed on screen for the whole trial. After each trial, the participants were presented with feedback confirming whether each goal was achieved or failed. After the experiment
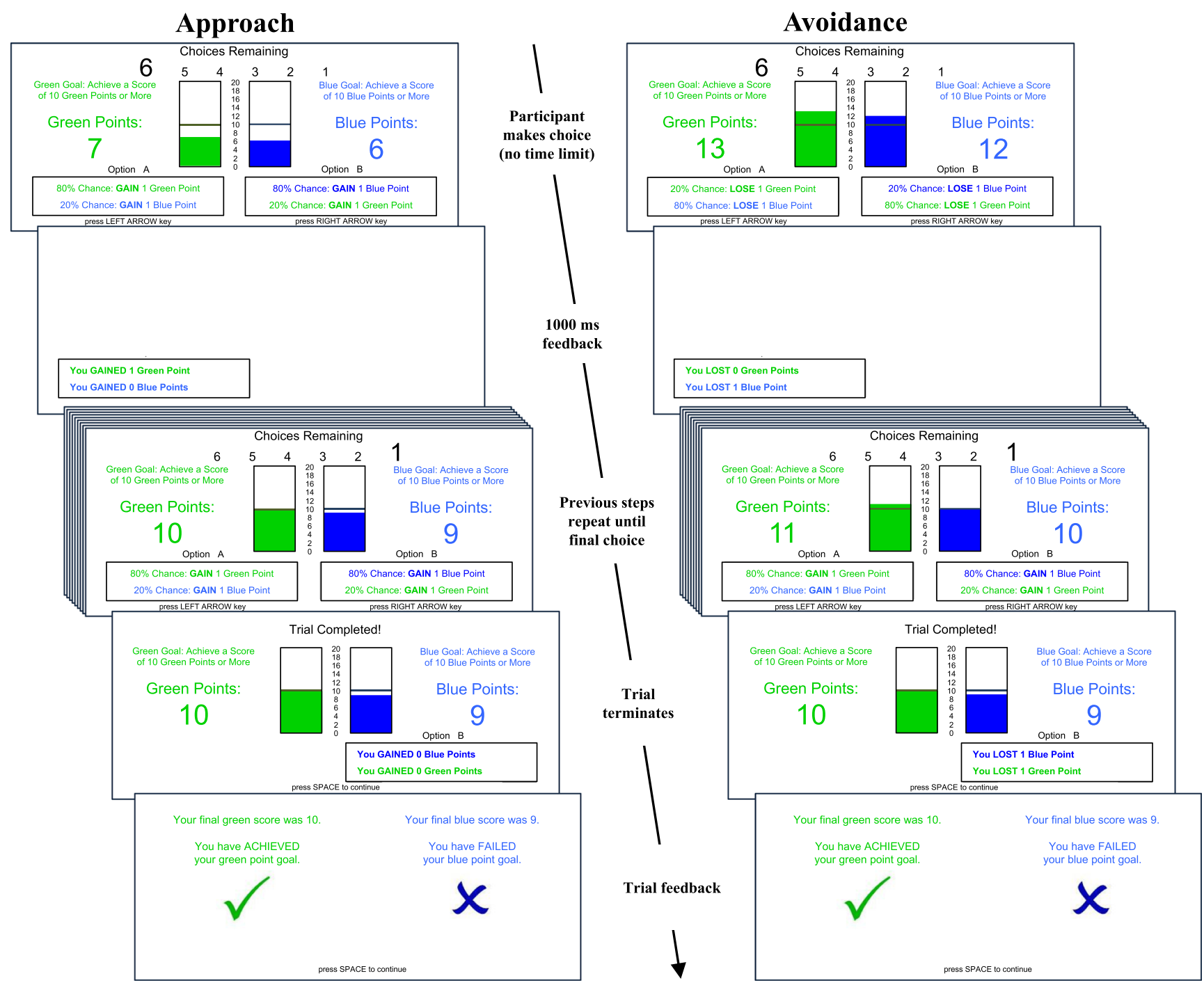

Fig. 1 Flow diagram of approach and avoidance trials 
was completed, each participant received the monetary incentive. The experiment took approximately $60 \mathrm{~min}$ to complete. The total number of decisions made across all participants was 62,370 (770 decisions per participant x 81 participants).

\section{Results}

Participants achieved both goals on $28 \%$ of trials, only one goal on $60 \%$ of trials, and neither goal on $12 \%$ of trials. As expected, people achieved only one goal more often as the incentive for achieving one goal increased (50\% of trials in the $\$ 0$ condition compared to $67 \%$ in the $\$ 10$ condition). We examined a) the influence of incentive structure and frame on prioritization and $b$ ) the subjective value of goal achievement. We first present results regarding the effects of incentive structure and frame and then present results regarding the subjective value of various goal achievement outcomes. In the experiment, the theoretically diagnostic decisions were those in which the two goals did not have equal scores (in cases where the blue and green points were equal, the choice was arbitrary) and where neither goal had already been achieved or failed. We excluded all other decisions from the analysis. The total number of decisions that remained across all participants was 28,203 .

\section{The effects of incentive structure and frame on prioritization}

We tested the effects of incentive structure and frame by comparing a series of Bayesian logistic mixed effects models using the blme package (Chung et al., 2013) in R. In each model, the dependent variable was whether or not the selected option prioritized the color with fewer points (i.e., the more difficult goal; $1=$ yes, $0=$ no). Each model included the random effects of trial and participant. We examined the evidence for the incentive effect by comparing the evidence for a model that included the fixed effect of the incentive for achieving one goal with a null model that did not include any fixed effects. We examined the evidence for the goal frame effect by comparing the evidence for a model that included the only fixed effect of frame with the null model. Finally, we examined the evidence for the incentive $\times$ goal frame interaction by comparing the evidence for a model that included the fixed effects of incentive, goal frame, and the interaction with a model that only included the fixed effects of incentive and goal frame.

Models were compared by calculating a Bayes factor (BF) for each pair. The BF indicates the relative evidence given by the data for Model 1 versus Model 2, and can be calculated based on the difference in Bayesian Information Criteria (BIC) between the two models:

$B F_{12}=\exp \left(-0.5 *\left(B I C_{1}-B I C_{2}\right)\right)$

$\mathrm{A} \mathrm{BF}$ of 1 indicates that the relative evidence for the two models is equal. A BF greater than 1 indicates evidence in favor of Model 1. Values between 1 and 3 can be regarded as weak evidence for Model 1, values between 3-20 as "positive" evidence, values between 20-150 as "strong" evidence, and values greater than 150 as "very strong" evidence (Kass \& Raftery, 1995; Wagenmakers, 2007).

The blme package by default imposes flat prior distributions on the fixed effects and noncommittal Wishart distributions on the variances and covariances of the random effects. We used these priors for the analyses. The BFs indicated very strong evidence for the incentive only and frame only models compared to the null model, and strong evidence for the interaction model over the model with main effects for incentive and frame (see Table 1). These results provide evidence for both main effects and the interaction. ${ }^{1}$ The tendency to prioritize the more difficult goal was generally higher when people pursued avoidance compared to approach goals (see Fig. 2). Furthermore, the tendency to prioritize the more difficult goal decreased as the incentive for achieving the first goal increased. ${ }^{2}$

\section{The subjective value of goal achievement}

To quantify the extent to which people discounted the value of achieving the first goal, we examined the subjective value of different goal achievement outcomes in each condition. We estimated these subjective values using a Bayesian parameter estimation approach which determined the value function that best describes participants' decisions. The value function was defined by a single parameter referred to as the subjective value ratio $(S V R)$. The $S V R$ represents the ratio of the value associated with achieving one goal to the value associated with achieving both goals. We assumed that $S V R$ could fall between 0 and 1 . An $S V R$ of 0 indicates that the participant behaved as if they placed no value on achieving only one goal, whereas an $S V R$ of 1 indicates that the participant behaved as if they placed as much value on achieving one goal as they placed on achieving both (i.e., they could not derive any additional value from achieving the second goal). Given a particular $S V R$, the expected values of each action under the reward maximizing model were

\footnotetext{
${ }^{1}$ These results were subjected to prior sensitivity analyses, which showed that the substantive interpretation of the effects was robust to the choice of prior.

${ }^{2}$ Because choices made in later stages of a trial depend on choices made previously in the trial, we examined whether the effects shown in Fig. 2 changed when only the first decision in each trial was analyzed. The results were broadly similar across the two analyses.
} 
Table 1 Fixed effect posterior modes for incentive, frame, and incentive $\mathrm{X}$ frame interaction in each model tested

\begin{tabular}{|c|c|c|c|c|c|}
\hline Model & Intercept & Incentive & Frame & Frame $\mathrm{X}$ incentive & Bayes factor \\
\hline Null model & 0.50 & - & - & - & \\
\hline Incentive only model & 1.31 & -0.15 & - & & $>10^{5}$ \\
\hline Frame only model & 0.51 & - & -0.40 & - & $>10^{5}$ \\
\hline Main effects model & 0.14 & -0.16 & -0.40 & - & \\
\hline Interaction model & 1.34 & -0.16 & -0.31 & -0.02 & 66.33 \\
\hline
\end{tabular}

Note: The BFs shown in the table reflect the difference between that model and the next simplest model

calculated using dynamic programming (e.g., Ballard et al., 2016). The difference between the expected valuesof the two actions were then transformed into a choice probability using a logistic function:

$$
p_{\text {blue }}=\frac{1}{1+\exp \left[-\tau\left(e v_{\text {blue }}-e v_{\text {green }}\right)\right]}
$$

where $e v_{\text {blue }}$ and $e v_{\text {green }}$ are the expected values of selecting the option that prioritizes the blue and green goal, respectively, $\tau$ is a parameter that controls variability in responding. When $\tau=0$, responses are completely random. As $\tau$ increases, responses become more deterministic.

Posterior distributions on parameters were obtained using JAGS (Plummer, 2003). Three parameters were estimated for each participant: an $S V R$ for the approach condition $\left(S V R_{a p}\right)$, an $S V R$ for the avoidance condition $\left(S V R_{a v}\right)$, and the $\tau$ parameter. The prior distributions for all three parameters were uninformative. The prior on the $S V R$ parameters was a uniform distribution that was bounded at 0 and 1 . The prior on $\tau$ parameter was a uniform distribution that was bounded at 0 and 500, which covered the range of plausible parameter values.

Figure 3 shows the average posterior medians for the $S V R_{a p}$ and $S V R_{a v}$ parameters, the value ratio under a reward maximizing model, and the individual credibility intervals, for each incentive condition. In both approach and avoidance conditions the $S V R$ tended to increase as the incentive for achieving one goal increased. Accordingly, the higher the monetary value attached to the completion of a single goal, the more it was valued. However, this increase was weaker than the corresponding increase in value ratio under a reward maximizing model (dashed lines),

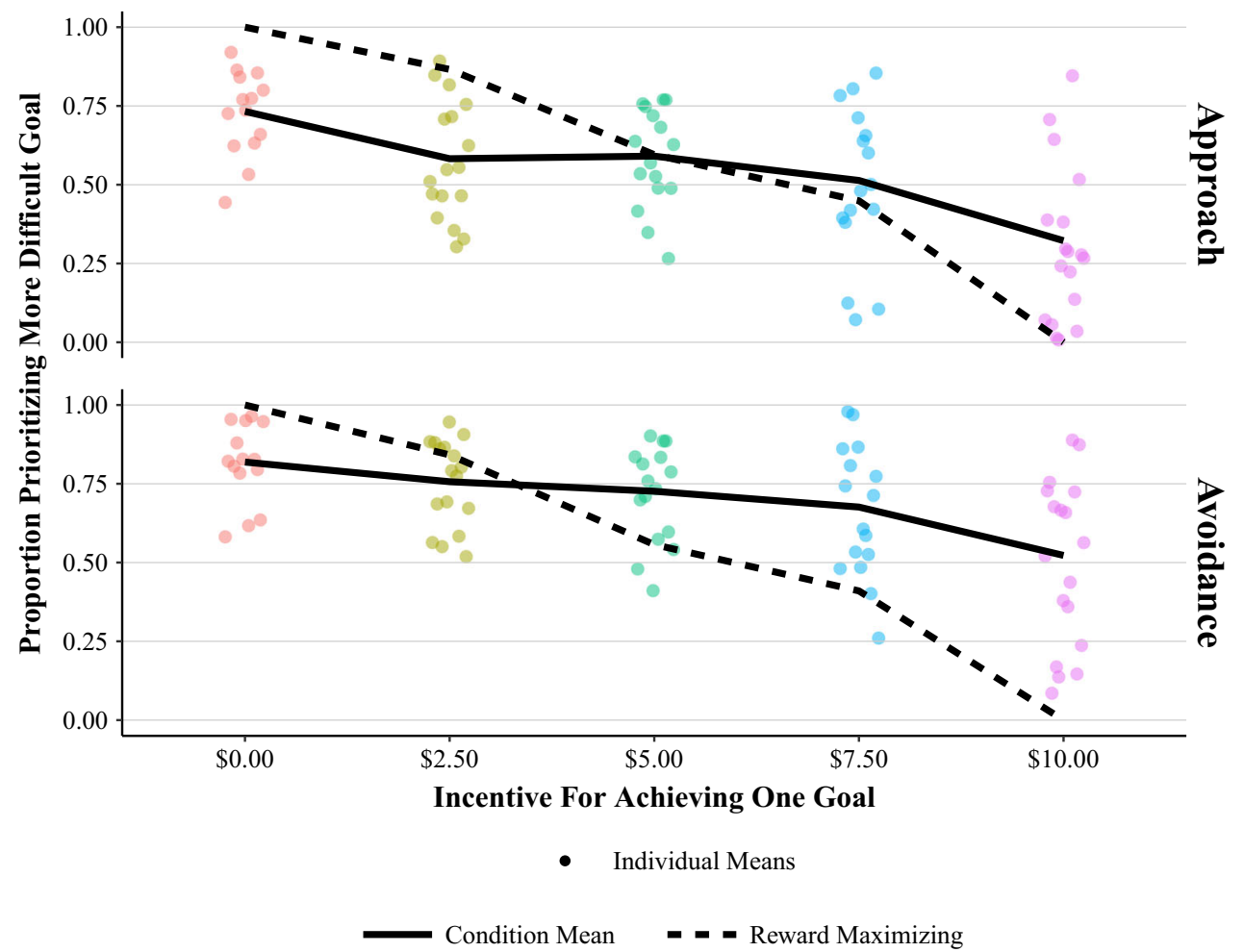

Fig. 2 Proportion of choices prioritizing the more difficult goal as a function of the incentive for achieving one goal and goal frame 


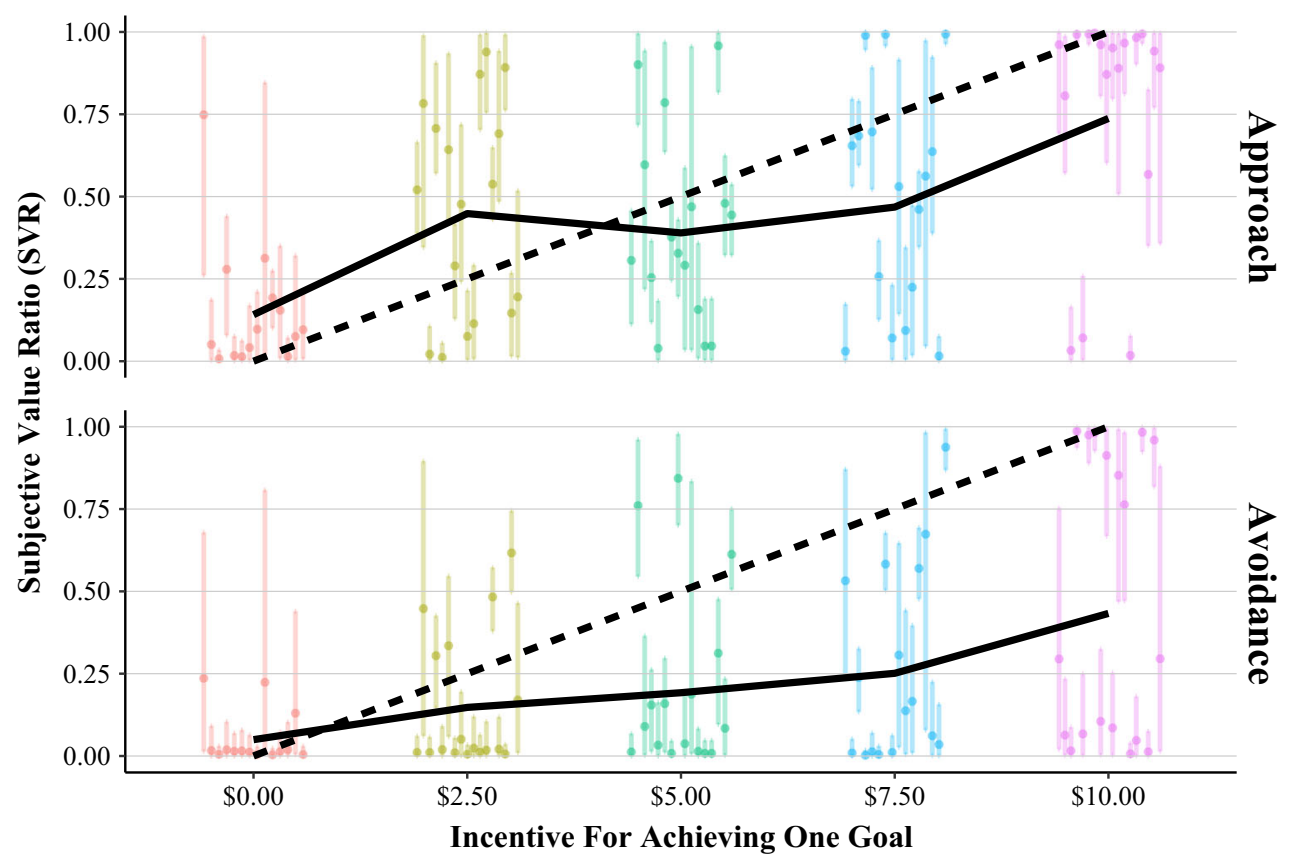

- Individual Medians (with 95\% CI)

Condition Median

- - Reward Maximizing

Fig. 3 Posterior median SVRs as a function of the incentive for achieving one goal and goal frame. The error bars represent the $95 \%$ credible intervals

particularly in the avoidance condition. The gap between the $S V R$ and the reward-maximizing value ratio indicates that the participants were generally placing less value on achieving one goal (and therefore more value on achieving both goals) than would be expected under a reward maximizing model, especially when the incentive for achieving one goal is higher. For example, in the $\$ 10.00$ condition, participants received the full reward after achieving the first goal, but only behaved as if achieving the first goal is about $60 \%$ (in approach) or $40 \%$ (in avoidance) as valuable as achieving both goals. In the $\$ 7.50$ condition, participants received $75 \%$ of the reward after achieving the first goal, but only behaved as if this outcome was about $33 \%$ as valuable as achieving both goals.

Although the average $S V R$ distributions provided evidence that participants were discounting the value of achieving the first goal, inspection of the individual credibility intervals suggests that there were individual differences in goal completion preferences. These individual differences were especially apparent in the approach condition. In this condition, many participants had $S V R$ credibility intervals that were entirely below the reward-maximizing value ratio, suggesting that they discounted the achievement of the value of achieving the first goal. A smaller number of participants in the approach condition had credibility intervals that were entirely above the reward-maximizing value ratio, suggesting that they over-valued the achievement of the first goal. Finally, some participants had $S V R$ credibility intervals that overlapped with the value ratio under the reward-maximizing model, suggesting that their behavior aligned with reward maximization. These individual differences were less apparent in the avoidance condition, however, where the majority of participants' credibility intervals were below the reward-maximizing value ratio. These findings reinforce the notion that the tendency to discount the achievement of the first goal was stronger in the avoidance condition.

\section{Discussion}

Our aim was to examine whether people discount the value of achieving the first goal when simultaneously pursuing multiple goals. We used Bayesian parameter estimation in a dynamic programming model to quantify the subjective value participants placed on various goal achievement outcomes. This analysis indicated that although people were sensitive to the incentives, they often departed from rewardmaximization by placing less value on the completion of a single goal than would be expected under a reward maximizing model. This finding suggests that people often discount the achievement of the first goal, and therefore may forego financial reward in order to achieve both task goals. 
The pattern of discounting depended on whether people were faced with approach or avoidance goals. Consistent with previous work (Ballard et al., 2016; Ballard et al., 2016), people prioritized the more difficult goal more often when pursuing avoidance goals than approach goals. We also found that the extent to which people discount the value of the first goal was stronger when pursuing avoidance goals. In this condition in particular, people exhibited a strong preference for attempting to achieve both goals. This preference was so strong that even when the full reward could be obtained after a single goal was achieved, people still prioritized the more difficult goal more than half the time.

These findings suggest that people are not always willing to accept smaller monetary rewards for less work (i.e., effort discounting). People may demonstrate the opposite tendency when a task has explicit goals. By working to achieve multiple goals even when most or all of the reward can be obtained after the first is achieved, people expended more resources than were required to obtain a reward. We believe this tendency exists because goal achievement has psychological value, which motivates effort even when the goal does not carry any objective reward (Brunstein, 1993; Heath et al., 1999; Sheldon \& Kasser, 1998; Tubbs, 1986; Wang \& Johnson, 2012). The findings underscore the importance of taking into account task goals when attempting to understand the relationship between effort and monetary reward.

Our findings suggest that the psychological value of goal achievement may differ across individuals. Many participants discounted the value of achieving the first goal relative to the reward-maximizing model, but some valued it more strongly. The psychological value of goal achievement may also depend on the balance between extrinsic and intrinsic motivators in the task. For example, if the incentives in the task were increased tenfold, the psychological value of the goals themselves would likely become relatively small, and behavior would more closely align with reward maximization (Gneezy \& Rustichini, 2000). By contrast, when an individual is pursuing goals that are more intrinsically rewarding, the psychological value would likely increase, and the financial rewards would become less influential.

More broadly, this research demonstrates the importance of goals for understanding decision making. Many studies in the decision making literature focus on static, one-shot decisions which differ markedly from the types of environments to which we might ultimately seek to generalize. The types of decisions people commonly face, like the trade-offs faced by the retail workers and academics in our opening examples, require the person to consider different types of goals, deadlines, and other environmental dynamics. We hope that this work helps to create a bridge between basic decision making research and the motivation and goal pursuit literatures that enhances our understanding of how these processes interact.

\section{Appendix}

Table 2 Unique trial configurations

\begin{tabular}{lll}
\hline $\begin{array}{l}\text { Number of } \\
\text { decisions }\end{array}$ & $\begin{array}{l}\text { Starting scores } \\
\text { (approach) }\end{array}$ & $\begin{array}{l}\text { Starting scores } \\
\text { (avoidance) }\end{array}$ \\
\hline 6 & 8,7 & 14,13 \\
6 & 8,6 & 14,12 \\
6 & 8,5 & 14,11 \\
6 & 8,4 & 14,10 \\
6 & 7,6 & 13,12 \\
6 & 7,5 & 13,11 \\
6 & 7,4 & 13,10 \\
5 & 8,7 & 13,12 \\
5 & 8,6 & 13,11 \\
5 & 8,5 & 13,10 \\
4 & 9,8 & 13,12 \\
4 & 8,7 & 12,11 \\
4 & 8,6 & 12,10 \\
3 & 9,8 & 12,11 \\
3 & 9,7 & 12,10 \\
2 & 9,8 & 11,10 \\
\hline
\end{tabular}

Note: Corresponding starting score pairs for the approach and avoidance conditions are equivalent in difficulty

\section{References}

Ballard, T., Yeo, G., Loft, S., Vancouver, J. B., \& Neal, A. (2016). An integrative, formal model of motivation and decision making: The MGPM*. Journal of Applied Psychology, 101, 1240-1265. doi:10.1037/apl0000121

Ballard, T., Yeo, G., Neal, A., \& Farrell, S. (2016). Departures from optimality when pursuing multiple approach or avoidance goals. Journal of Applied Psychology, 101, 1056-1066. doi:10.1037/apl0000082

Baumeister, R. F., Bratslavsky, E., Finkenauer, C., \& Vohs, K. D. (2001). Bad is stronger than good. Review of General Psychology, 5, 323-370. doi:10.1037/1089-2680.5.4.323

Botvinick, M., \& Braver, T. (2015). Motivation and cognitive control: From behavior to neural mechanism. Annual Review of Psychology Psychol, 66, 83-113. doi:10.1146/annurev-psych-010814-015044

Botvinick, M., Huffstetler, S., \& McGuire, J. T. (2009). Effort discounting in human nucleus accumbens. Cognitive, Affective, \& Behavioral Neuroscience, 9, 16-27. http://www.sciencemag.org/ content/306/5703/1895.short. doi:10.3758/CABN.9.1.16.Effort

Brunstein, J. C. (1993). Personal goals and subjective well-being: A longitudinal study. Journal of Personality and Social Psychology, $65,1061-1070$. 
Chong, T. T. J., Bonnelle, V., Manohar, S., Veromann, K. R., Muhammed, K., Tofaris, G. K., \& Husain, M. (2015). Dopamine enhances willingness to exert effort for reward in Parkinson's disease. Cortex, 69, 40-46. doi:10.1016/j.cortex.2015.04.003

Chung, Y., Rabe-Hesketh, S., Dorie, V., Gelman, A., \& Liu, J. (2013). A nondegenerate penalized likelihood estimator for variance parameters in multilevel models. Psychometrika, 78, 685-709.

Gneezy, U., \& Rustichini, A. (2000). Pay enough or don't pay at all. The Quarterly Journal of Economics, 115, 791-810.

Heath, C., Larrick, R. P., \& Wu, G. (1999). Goals as reference points. Cognitive Psychology, 38, 79-109. doi:10.1006/cogp.1998.0708

James, W. (1993). Rewards, experience and decision costs in first price auctions. Economic Inquiry, 31, 237.

Kahneman, D., \& Tversky, A. (1979). Prospect theory: An analysis of decision under risk. Econometrica, 47, 263-291.

Kass, R. E., \& Raftery, A. E. (1995). Bayes factors. Journal of the American Statistical Association, 90, 773-795.

Koo, M., \& Fishbach, A. (2008). Dynamics of self-regulation: How (un)accomplished goal actions affect motivation. Journal of Personality and Social Psychology, 94, 183-95. doi:10.1037/ 0022-3514.94.2.183

Kool, W., \& Botvinick, M. (2014). A labor/leisure tradeoff in cognitive control. Journal of Experimental Psychology: General, 143, 131141. doi:10.1037/a0031048

Kool, W., McGuire, J. T., Rosen, Z. B., \& Botvinick, M. M. (2010). Decision making and the avoidance of cognitive demand. Journal of Experimental Psychology: General, 139, 665-682. doi:10.1037/a0020198

Libedinsky, C., Massar, S. A. A., Ling, A., Chee, W., Huettel, S. A., \& Chee, M.W. L. (2013). Sleep deprivation alters effort discounting but not delay discounting of monetary rewards. Sleep, 36, 899904. doi: $10.5665 /$ sleep. 2720

Lord, R. G., Diefendorff, J. M., Schmidt, A. M., \& Hall, R. J. (2010). Self-regulation at work. Annual Review of Psychology, 61, 543568. doi:10.1146/annurev.psych.093008.100314

Louro, M. J., Pieters, R., \& Zeelenberg, M. (2007). Dynamics of multiple-goal pursuit. Journal of Personality and Social Psychology, 93, 174-193. doi:10.1037/0022-3514.93.2.174

Neal, A., Ballard, T., \& Vancouver, J. B. (2017). Dynamic selfregulation and multiple-goal pursuit. Annual Review of Organizational Psychology and Organizational Behavior, 4, 410-423. doi:10.1146/annurev-orgpsych-032516-113156

Plummer, M. (2003). JAGS: A program for analysis of Bayesian graphical models using Gibbs sampling. In Hornik, K., Leische, F., \& Zeileis, A. (Eds.) Proceedings of the 3rd International
Workshop on Distributed Statistical Computing (DSC 2003). Technische Universität Wien, Vienna, Austria.

Schmidt, A. M., \& DeShon, R. P. (2007). What to do? The effects of discrepancies, incentives, and time on dynamic goal prioritization. Journal of Applied Psychology, 92, 928-941. doi:10.1037/0021-9010.92.4.928

Schmidt, A. M., \& Dolis, C. M. (2009). Something's got to give: The effects of dual-goal difficulty, goal progress, and expectancies on resource allocation. Journal of Applied Psychology, 94, 678-691. doi:10.1037/a0014945

Schmidt, A. M., Dolis, C. M., \& Tolli, A. P. (2009). A matter of time: Individual differences, contextual dynamics, and goal progress effects on multiple-goal self-regulation. Journal of Applied Psychology, 94, 692-709. doi:10.1037/a0015012

Sheldon, K. M., \& Kasser, T. (1998). Pursuing personal goals: Skills enable progress, but not all progress is beneficial. Personality and Social Psychology Bulletin, 24, 1319-1331.

Shenhav, A., Botvinick, M. M., \& Cohen, J. (2013). The expected value of control: An integrative theory of anterior cingulate cortex function. Neuron, 79, 217-240. doi:10.1016/j.neuron.2013. 07.007

Smith, V. L. (1976). Experimental economics: Induced value theory. The American Economic Review, 66, 274-279.

Smith, V. L. (1982). Microeconomic systems as an experimental science. The American Economic Review, 72, 923-955.

Tubbs, M. E. (1986). Goal setting: A meta-analytic examination of the empirical evidence. Journal of Applied Psychology, 71, 474-483. doi:10.1037//0021-9010.71.3.474

Unsworth, K., Yeo, G., \& Beck, J. (2009). Multiple goals: A review and derivation of general principles. Journal of Organizational Behavior, 30, 839-862. doi:10.1002/job

Vancouver, J. B., Weinhardt, J. M., \& Schmidt, A. M. (2010). A formal, computational theory of multiple-goal pursuit: Integrating goal-choice and goal-striving processes. Journal of Applied Psychology, 95, 985-1008. doi:10.1037/a0020628

Wagenmakers, E. J. (2007). A practical solution to the pervasive problems of $p$ values. Psychonomic Bulletin \& Review, 14, 779804.

Wang, X. T., \& Johnson, J. G. (2012). A tri-reference point theory of decision making under risk. Journal of Experimental Psychology: General, 141, 743-756. doi:10.1037/a0027415

Westbrook, A., Kester, D., \& Braver, T. S. (2013). What is the subjective cost of cognitive effort? Load, trait, and aging effects revealed by economic preference. PLOS ONE, 8, 1-8. doi:10.1371/journal. pone. 0068210 